\title{
Can palmar creases serve as landmarks for the deeper neuro-vascular structures?
}

\author{
M. Kwiatkowska · T. Jakutowicz $\cdot$ B. Ciszek $\cdot$ J. Czubak
}

Received: 1 July 2013 / Accepted: 26 September 2013 / Published online: 11 October 2013

(C) The Author(s) 2013. This article is published with open access at Springerlink.com

\begin{abstract}
Purpose The aim of this study was the examination of the superficial anatomy of palmar creases and their relation to deeper neuro-vascular structures.

Methods Four creases: distal wrist flexion crease, thenar crease, proximal palmar crease and distal palmar crease were evaluated with reference to the following structures: palmar cutaneous branch of median nerve, palmar cutaneous branch of ulnar nerve, the nerve of Henle, transverse palmar branches from ulnar nerve, recurrent motor branch of median nerve, radial proper palmar digital nerve to the index and the ulnar proper palmar digital nerve to the thumb, Berrettini's communicating branch, ulnar nerve and artery, superficial palmar arch. We performed dissections of 20 cadaveric upper limbs derived from a homogenous Caucasian group. In our study we measured the location of surgically important structures with reference to palmar skin creases.

Results Among the other observations we noticed that the palmar cutaneous branches of the median and ulnar nerves were located at least $0.5 \mathrm{~cm}$ away from the thenar crease.
\end{abstract}

We declare that the experiments comply with the current laws of the country in which they were performed (i.e. Polish law).

M. Kwiatkowska $\cdot$ B. Ciszek

Department of Descriptive and Clinical Anatomy, Centre of Biostructure, Research Medical University of Warsaw, Chalubinskiego 5, 02-004 Warsaw, Poland

e-mail: magdkwiat@gmail.com

M. Kwiatkowska · T. Jakutowicz $(\bowtie) \cdot$ J. Czubak Department of Orthopaedics, Paediatric Orthopaedics and Traumatology, The Medical Centre of Postgraduate Education, Gruca Teaching Hospital, Konarskiego 13, Otwock, 05-400 Warsaw, Poland

e-mail: tjakutowicz@o2.pl
The superficial palmar arch was found between the thenar and proximal palmar crease and never crossed the proximal or distal palmar creases.

Conclusions These anatomical dissections will provide reference material for further ultrasound studies on the arrangements of neuro-vascular structures in reference to superficial palmar creases.

Keywords Palmar creases $\cdot$ Hand anatomy $\cdot$ Wrist anatomy · Palmar cutaneous branch of median nerve . Palmar cutaneous branch of ulnar nerve $\cdot$ Superficial palmar arch $\cdot$ Berrettini's communicating branch

\section{Introduction}

The palmar creases have been the subject of interest to scientists for centuries. Nevertheless, there are very few current references addressing the anatomy of palmar creases and its clinical significance. So far in the literature there have been descriptions of the palmar creases in reference to osseous structures or genetically inherited disorders $[5,7$, 28]. Although the surgical approach to the palmar surface of the hand is usually straightforward, it may be occasionally a challenging task. There are several small cutaneous branches of nerves that are at risk when approach is being made to operate the hand. Several different incisions have been proposed to avoid the damage of these structures [1, $3,6,10,16,21,24,25,29,33]$. In our study for the first time we decided to use the natural palmar lines-creases as a guide to localise precisely the important neuro-vascular structures.

The aim of this study was to examine the superficial anatomy of palmar creases and their relation to deeper structures. Four palmar creases were evaluated: 


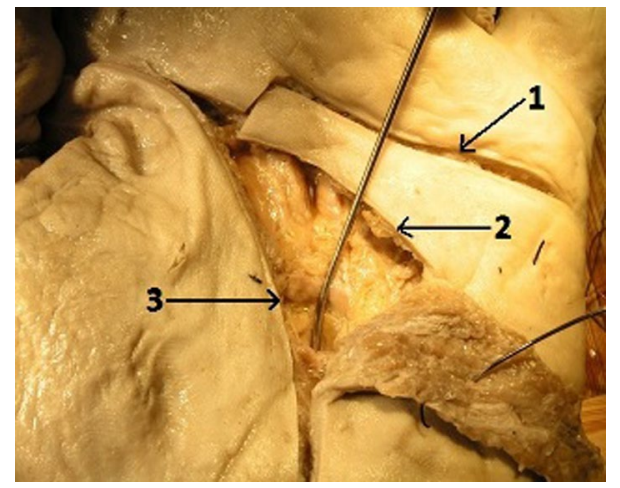

Fig. 1 Palmar surface of the left hand. Incisions following the natural palmar creases: 1 distal palmar crease, 2 proximal palmar crease, 3 thenar crease. The probe is inserted deep to the flexor retinaculum

distal wrist flexion crease, thenar crease, proximal palmar crease and distal palmar crease. The anatomy of palmar skin creases was evaluated with reference to the following structures: palmar cutaneous branch of median nerve (PCBMN), palmar cutaneous branch of ulnar nerve (PCBUN), the nerve of Henle, transverse palmar branches from ulnar nerve, recurrent motor branch of median nerve, the ulnar proper palmar digital nerves to the thumb, the radial proper palmar digital nerve to the index, Berrettini's communicating branch, ulnar nerve and artery and superficial palmar arch.

\section{Materials and methods}

We performed dissections of 20 cadaveric upper limbs (11 right and 9 left). The specimens were obtained from the Department of Anatomy at the Medical University of Warsaw, Poland. Twelve limbs were fixed with formaldehyde solution and eight were fresh frozen. All specimens were derived from a homogenous Caucasian group. The dissections of the palmar surface of hands were performed with the aid of surgical microscope with magnification $2-5.5 \times$. The incisions were performed within the natural palmar creases from the proximal to the distal end of the crease (Fig. 1). In the case of the distal wrist creases and the distal palmar creases the dissection was begun from the ulnar side of the crease. The subcutaneous fat was very thin under these creases and made the beginning of dissection much easier. In the first step of our work we were able to separate the palmar skin from the underlying subcutaneous fat leaving this layer intact. This was essential for cutaneous nerves identification and preservation during dissection. The palmar creases and the deeper structures, listed above, were identified and documented with a digital camera (Konica Minolta Dimage A200 8MP with Anti-shake $7 \times$ optical zoom, Korea) and analysed using a computer

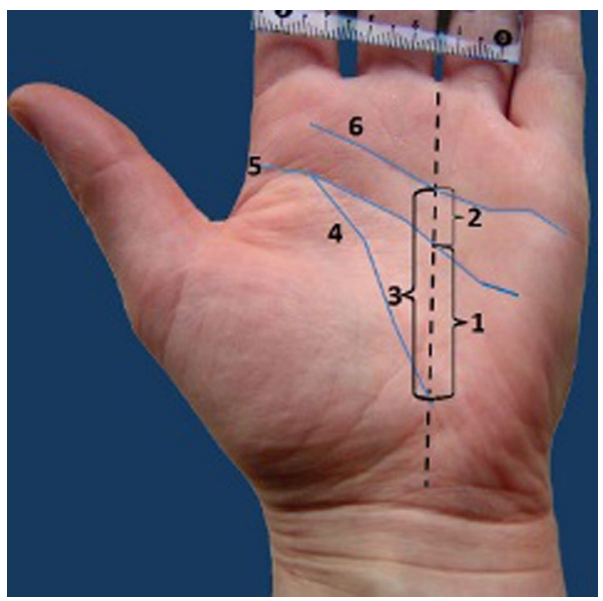

Fig. 2 Distances between the creases. Measurements performed along the radial axis of the ring finger. 1 Distance between the thenar crease and the proximal palmar crease, 2 distance between proximal and distal palmar crease, 3 distance between the thenar and distal palmar crease, 4 thenar crease, 5 proximal palmar crease, 6 distal palmar crease

system (MultiScanBase v.13.08; Computer Scanning System, Warsaw, Poland). The measurements were taken from the photographs of the dissected specimens. Each picture contained a $5-\mathrm{cm}$-long ruler. The scale tools for the measurements were manually calibrated. The distances from the thenar crease to the proximal palmar crease and the distal palmar crease were measured along the radial axis of the ring finger (Fig. 2). The measurements of the structures crossing the distal wrist crease were performed from the ulnar to the radial side of the hand. The results of measurements were automatically transferred to the database of the MultiScanBase.

\section{Results}

\section{Thenar crease}

The thenar crease usually intersects the lateral side of the proximal palmar crease and curves obliquely across the palm to intersect the distal wrist crease near the wrist centre. The mean length of thenar crease was $7.37 \mathrm{~cm}$ (range 5-10.7 cm). The mean distance from thenar crease to proximal and to distal palmar crease evaluated along the radial axis of the ring finger (Fig. 2) was $0.97 \mathrm{~cm}$ (range $0.7-1.3 \mathrm{~cm}$ ) and $1.58 \mathrm{~cm}$ (range $1.2-2.1 \mathrm{~cm}$ ), respectively.

The thenar crease, in our dissections, was never crossed by any cutaneous or motor branches of nerves. These structures were always located a few millimetres away from the thenar creases (at least $5 \mathrm{~mm}$ ). The only structure to be found within the course of the thenar crease is the carpal tunnel (Table 1; Figs. 1, 3). 
Table 1 Mean distances from thenar crease to important neuro-vascular structures of the wrist

\begin{tabular}{lllll}
\hline $\begin{array}{l}\text { Palmar cutaneous } \\
\text { branch of the ulnar } \\
\text { nerve (PCBUN) }\end{array}$ & $\begin{array}{l}\text { Palmar cutaneous } \\
\text { branch of the median } \\
\text { nerve (PCBMN) }\end{array}$ & $\begin{array}{l}\text { Recurrent branch of } \\
\text { the median nerve }\end{array}$ & $\begin{array}{l}\text { Branch of the median } \\
\text { nerve to the index }\end{array}$ & $\begin{array}{l}\text { Ulnar nerve, artery } \\
\text { and the nerve of } \\
\text { Henle }\end{array}$ \\
\hline $2.12 \mathrm{~cm}(0.7-3)$ & $1.1 \mathrm{~cm}(0.7-1.4)$ & $1.09 \mathrm{~cm}(0.6-2.3)$ & $1.87 \mathrm{~cm}(0.5-3.5)$ & $1.71 \mathrm{~cm}(1-2.3)$ \\
\hline
\end{tabular}

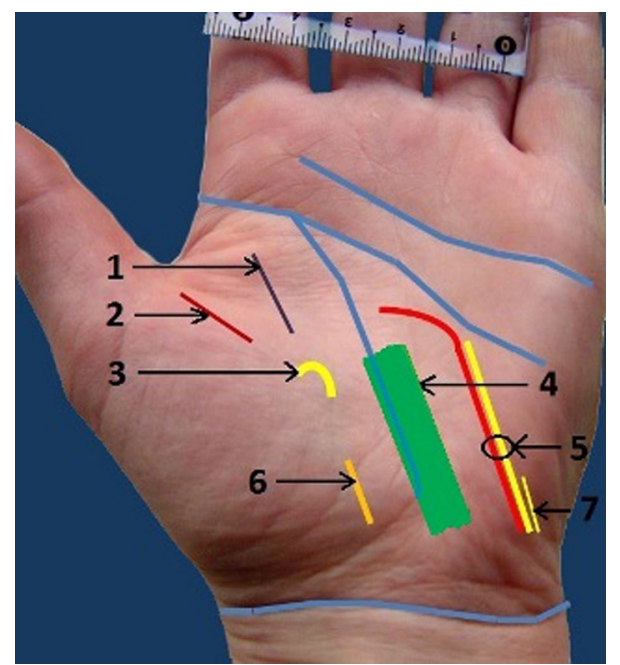

Fig. 3 Distances between creases, nerves, and arteries: 1 radial proper palmar digital nerve to the index, 2 ulnar proper palmar digital nerve to the thumb, 3 recurrent motor branch of the median nerve, 4 carpal tunnel, 5 ulnar nerve, artery and nerve of Henle, 6 palmar cutaneous branch of the median nerve (PCBMN), 7 palmar cutaneous branch of the ulnar nerve (PCBUN)

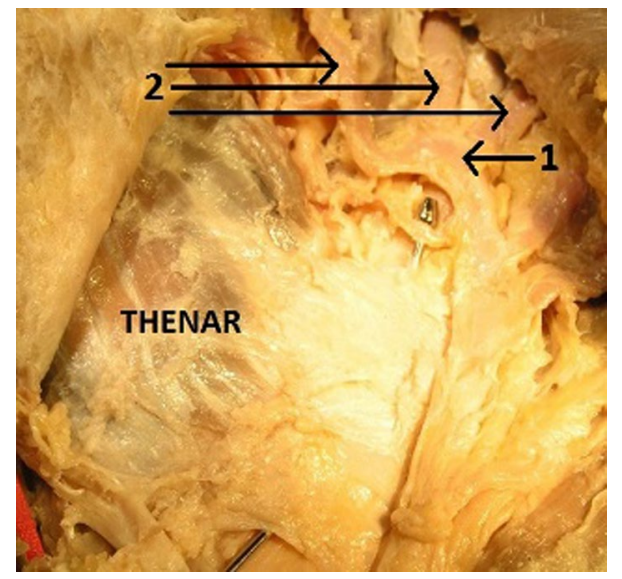

Fig. 4 Dissected palm of the left hand: 1 superficial palmar arch, 2 common palmar digital arteries. The probe is inserted under the flexor retinaculum

\section{Superficial palmar arch}

The superficial palmar arch (Fig. 4) is located just beneath the palmar fascia and on top of the superficial tendons and may be complete or incomplete. It is located between
Table 2 Distance from the superficial palmar arch to the thenar crease measured in three points of the arch

\begin{tabular}{ll}
\hline Distal part of the arch & $0.51 \mathrm{~cm}(0.02-1)$ \\
Convex part of the arch & $0.72 \mathrm{~cm}(0.4-1.2)$ \\
Proximal part of the arch & $1.07 \mathrm{~cm}(0.8-1.7)$ \\
\hline
\end{tabular}

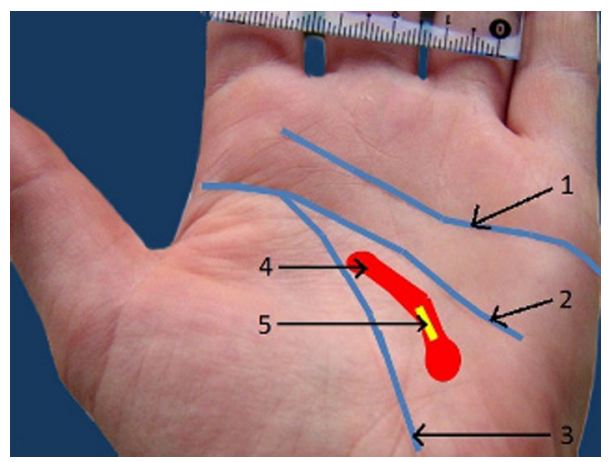

Fig. 5 Distance from palmar creases to the superficial palmar arch and Berrettini's communicating branch: 1 distal palmar crease, 2 proximal palmar crease, 3 thenar crease, 4 superficial palmar arch, 5 Berrettini's communicating branch

thenar crease and proximal palmar crease. The distance from superficial palmar arch to the thenar crease was measured in three points of the arch: radially to the thenar crease (distal part of the arch), in the max convex of the arch, ulnarly to the crease (proximal part of the arch). The superficial palmar arch never crossed the level of proximal palmar crease. Moreover, the Berrettini's communicating branch was located along the course of the superficial palmar arch and was usually located deep to the arch. In our study the superficial palmar arch was located between the thenar and the proximal palmar creases (Table 2; Fig. 5).

\section{Berrettini's communicating branch}

The communicating branch between the median and ulnar nerve, known as the Berrettini's communicating branch (Fig. 6), was also examined. This branch courses from the common digital nerve in the fourth interosseous space (ulnar nerve origin) to the common digital nerve in the third interosseous space (median nerve origin). Its course often parallels the superficial palmar arterial arch. In two cases this nerve was present in a plexiform nature. This 


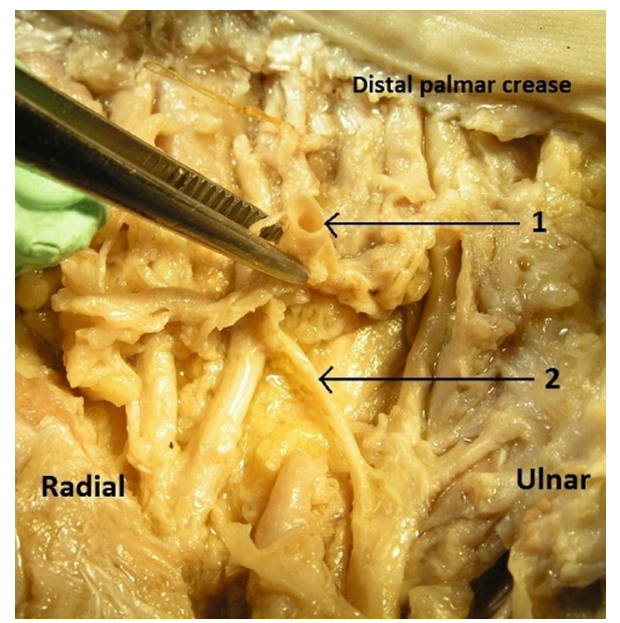

Fig. 6 Dissected palm of the left hand: 1 superficial palmar arch, cut, 2 Berrettini's communicating branch 3 distal palmar crease

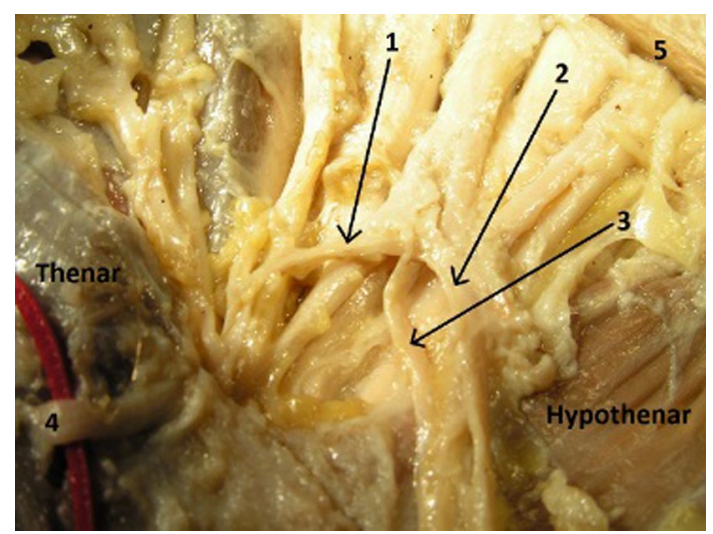

Fig. 7 Dissected palm of the left hand: 1 Berrettini's communicating branch, 2 ulnar nerve, 3 ulnar artery, 4 recurrent branch of median nerve, 5 distal palmar crease

communicating branch was perforated by the superficial palmar arch (Fig. 7). In the other specimens the Berrettini's communicating branch of ulnar nerve was located deep to the superficial palmar arch.

The length of the branch was an average of $0.94 \mathrm{~cm}$ (range $0.8-1.2 \mathrm{~cm}$ ). The distance from the thenar crease to the communicating branch was measured at two points:

1. At the ulnar end of the nerve.

2. At the median end of the nerve.

The superficial palmar arch and the Berrettini's communicating branch were always found between the thenar crease and the proximal palmar crease (Table 3; Fig 5). These structures never crossed the line of the proximal palmar crease.
Table 3 Distance from the thenar crease to the Berrettini's branch measured from both ends of the branch

\begin{tabular}{ll}
\hline From ulnar end & $1.1 \mathrm{~cm}(0.7-1.3)$ \\
From median end & $0.74 \mathrm{~cm}(0.4-1.3)$ \\
\hline
\end{tabular}

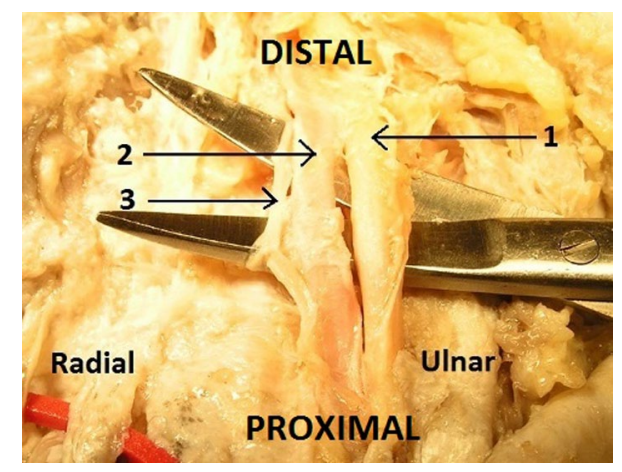

Fig. 8 Dissected palm of the left hand: 1 ulnar nerve, 2 ulnar artery, 3 nerve of Henle

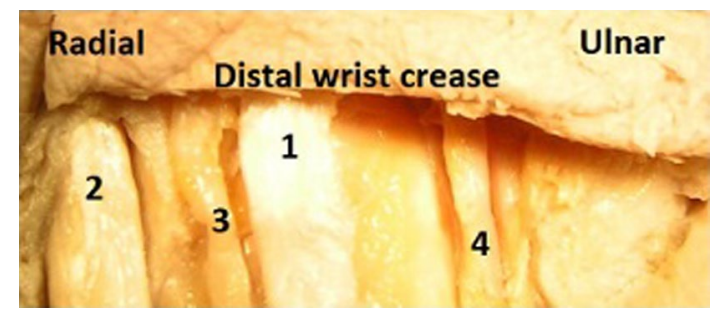

Fig. 9 Palmar surface of the left hand. Dissection follows the distal wrist crease: $1 P L$ palmaris longus tendon, $2 F C R$ flexor carpi radialis tendon, 3 PCBMN palmar cutaneous branch of the median nerve, 4 PCBUN palmar cutaneous branch of the ulnar nerve

\section{Nerve of Henle}

The triad of ulnar artery, nerve and nerve of Henle (Fig. 8) was also evaluated with reference to thenar crease.

The distance of this triad to thenar crease was an average of $1.71 \mathrm{~cm}$ (range $1-2.3 \mathrm{~cm}$ ). The nerve of Henle, the sympathetic nervi vasorum of the ulnar artery, gives additional sensory innervations to the palm. The nerve lies alongside the artery and then branches off into smaller nerves alongside the superficial palmar arch.

\section{Distal wrist flexion crease}

Although there are usually three wrist flexion creases, only the distal crease is of sufficient consistency to be used as a reliable landmark. The length of distal wrist crease was an average of $5.95 \mathrm{~cm}$ (range $4-7.5 \mathrm{~cm}$ ). 
The location of anatomical structures crossing the distal wrist crease and their arraignment was also evaluated (Fig. 9).

Flexor carpi radialis tendon was located an average of $1.4 \mathrm{~cm}$ (range $0.7-2.1$ ) from radial side of the hand crossing the line of the distal flexion wrist crease. Flexor carpi ulnaris tendon was identified an average of $1.35 \mathrm{~cm}$ (range $1.3-1.4 \mathrm{~cm}$ ) from ulnar side of the hand. Palmaris longus tendon was absent in two cases and when present located an average of $2.95 \mathrm{~cm}$ (range $2.6-3.3 \mathrm{~cm}$ ) from ulnar side of the hand and an average of $2.9 \mathrm{~cm}$ (range $2.4-3.3 \mathrm{~cm}$ ) from radial side.

Ulnar artery and nerve with nerve of Henle was detected an average of $1.6 \mathrm{~cm}$ (range $1.2-1.8 \mathrm{~cm}$ ) from ulnar side of the hand. The nerve of Henle was present in $100 \%$ of specimens.

PCBMN was located an average of $3.11 \mathrm{~cm}$ (range 2.6$3.7 \mathrm{~cm}$ ) away from radial side of the hand.

PCBUN was located an average of $3.82 \mathrm{~cm}$ (range $2-5.8 \mathrm{~cm}$ ) away from radial side of the hand.

The proximal and distal palmar creases

The length of proximal palmar crease was an average of $3.54 \mathrm{~cm}$ (range $0.7-7.7 \mathrm{~cm}$ ) and the length of distal palmar crease was of an average of $4.42 \mathrm{~cm}$ (range $1.3-7.0 \mathrm{~cm}$ ).

We also measured the distance from proximal to distal palmar crease along the radial axis of the ring finger and it was an average of $0.87 \mathrm{~cm}$ (range $0.6-1.0 \mathrm{~cm}$ ) (Fig. 2).

We evaluated the distance from superficial palmar arch and Berrettini's communicating branch to proximal and distal palmar creases.

The distance from superficial palmar arch to proximal palmar crease was an average of $1.33 \mathrm{~cm}$ (range $0.6-1.7 \mathrm{~cm}$ ) and to the distal palmar crease an average of $2.17 \mathrm{~cm}$ (range 1.9-2.7 cm).

The distance from the Berrettini's communicating branch to the proximal palmar crease was an average of $1.35 \mathrm{~cm}$ (range $0.6-2.1 \mathrm{~cm}$ ) and to the distal palmar crease an average of $2.21 \mathrm{~cm}$ (range 1.6-2.7 cm).

\section{Discussion}

Palmar creases, which are defined as the skin histologically attached to an underlying structure, are formed during the foetal period. The main creases of the palm may be morphologically constant, but with regard to length and position, they vary from person to person. The palmar creases naturally grow and develop with hand. They are unique for every human being. Palmar creases form at about 12 foetal weeks, although spontaneous hand movement does not occur before 11.5 foetal weeks. Therefore, although foetal hand movement may influence the development of the creases, genetic factors are considered most important to their formation $[7,19]$. Our understanding of the creases and their values is yet incomplete. The gaps in our insight limit the possible interpretations and practical applications of the knowledge gained from the studies of various aspects of flexion creases.

Four palmar creases were evaluated in present study: distal wrist flexion crease, thenar crease, proximal palmar crease and distal palmar crease. These creases were consistently present in our specimens. We performed dissections of twenty cadaveric limbs from which twelve were fixed with formaldehyde solution and eight were fresh frozen. We are aware of the existence of some limitations to the present study. This anatomic study was performed in nonliving tissue and thus in vivo conditions could not be examined. Moreover, the cadaveric post mortem processing might have altered the neuro-vascular relationships. We do feel, however, that our sample of specimens preserved in two different conditions reflects the broad spectrum of patients encountered in a hand surgery practice.

In 1953, the most famous surgical landmark of hand known as the Kaplan's [15, 17] cardinal line was described and used worldwide. Kaplan's description of his landmark was a line "drawn from the apex of the interdigital fold between the thumb and index finger toward the ulnar side of the hand, parallel with the middle crease of the hand" The middle crease of the hand is the crease located between the distal palmar crease and the thenar crease and is also known in the literature as the proximal palmar crease [9].

As the later studies show, the line may be presented and used in four different ways by hand surgeons [32]. This may lead to the unintentional damage of important cutaneous branches as well as the vascular structures such as superficial palmar arch [2, 11, 14, 23].

The Kaplan's cardinal line has been used as a surface marker in various anatomical studies. Anand and Trzeciak [1] anatomically correlated the relationship of Kaplan's cardinal line to the superficial palmar arch. They found the superficial palmar arterial arch was located an average of 10.4 and $11.8 \mathrm{~mm}$ from the radial and ulnar borders of the ring finger with standard deviations of roughly $4 \mathrm{~mm}$ for each measurement.

In our study the superficial palmar arch was located between thenar crease and proximal palmar crease, which is similar to results of Anand and Trzeciak.

Other investigators, including Kaplan himself, have described numerous variations to the cardinal line [4, 16, $17,27]$. Current textbooks, even pinnacle works on surgical approaches, differ in their portrayal of Kaplan's cardinal line [13]. This ambiguity becomes important when one considers the complex neurovascular patterns in the volar hand [11, 14]. More importantly, since Kaplan's cardinal 
line has been described as representing the surface correlate of the motor branch of the median nerve [13, 27], deep branch of ulnar nerve [4, 16, 27], distal extent of the transverse carpal ligament [4] and the superficial palmar arch [4, $16,18,27]$, it becomes paramount to accurately define the landmark. We believe that palmar skin creases can be helpful here and serve as important additional superficial landmarks to guide the surgeon.

In our anatomical study we examined and measured not only the palmar creases but also important neuro-vascular structures such as nerve of Henle, transverse branches of ulnar nerve and Berrettini's communicating branch. The nerve of Henle was present in $100 \%$ of our specimens. According to the literature the nerve serves as the nervi vasorum of the ulnar artery, and gives innervations to the forearm and palm, in addition providing sympathetic innervations to the artery $[9,29]$. This nerve was traced along the ulnar artery and the superficial palmar arch and its branches: common palmar digital arteries.

Accidental damage to this nerve can occur especially in the case of iatrogenic injury of the superficial palmar arch. This is not a benign occurrence and can result in complex regional pain syndrome [31].

Multiple cutaneous nerves to the palm are noted from the ulnar nerve. Many of these nerves exited perpendicularly from the longitudinal direction of the ulnar nerve, thus prompting a description of them as transverse palmar cutaneous branches of the ulnar nerve. These nerves pierced the palmar carpal ligament to innervate the skin and subcutaneous tissue of the hypothenar eminence and midpalm, usually distal to that area innervated by either the nerve of Henle or the palmar cutaneous branch of ulnar nerve $[9,29$, 34].

The communicating branch of ulnar nerve, known as the Berrettini's communicating branch, connecting the third common palmar digital branch of the median nerve with the fourth common palmar and proper palmar digital branches of the ulnar nerve was present in $100 \%$ of our specimens. The earliest illustrations of the communicating branch between the digital branches of the median and ulnar nerves were the Berrettini drawings in 1741 [8]. Studies on the incidence of Berrettini anastomosis suggested that it should be considered a normal structure rather than an anatomical variation as its incidence was found to be over $80 \%[8,20,22]$.

In two cases this nerve was present in a plexiform nature. This Berrettini's communicating branch was perforated by the superficial palmar arch. The unusual relationship of Berrettini anastomosis with the superficial palmar arch is very rare, and knowledge about such a variation is important when performing carpal tunnel release, flexor tendon surgery, Dupuytren's fasciectomy and when dealing with arterial repairs and vascular graft applications in the hand [30].

Endoscopic and open carpal tunnel releases, even if performed meticulously, still carry the potential for complications. A survey of American Society for Surgery of the Hand (ASSH) members published in 1999 noted a myriad of injuries, including damage to the superficial palmar arch (SPA) in both types of releases [26]. The preciseness of the incision utilised is paramount in preventing iatrogenic injury and ensuring a good outcome. The limited visual field in CTR, more appreciable in the endoscopic method, attests to the importance of topographical markers to delineate underlying structures [12, 26, 31]. Incisions can then be planned taking into consideration potential areas of neurovascular vulnerability. Thus examination of the superficial skin markers can make it easier to plan the operation.

As we mentioned above our cadaveric study has some limitation, but in the future we are planning to expand it on living subjects by performing the ultrasound examination of palmar hand surface. We will try to localise ultrasonographically the neurovascular structures with their correlation to palmar skin creases.

We hope this future study will add to that anatomical one and will help us to use the palmar skin creases as the reliable topographical landmarks for planning the safe surgical incisions.

\section{Conclusions}

The palmar creases may serve as landmarks for the deeper neuro-vascular structures.

The superficial palmar arch was found between the thenar and proximal palmar crease and never crossed the proximal palmar crease.

The thenar crease, in our dissections, was never crossed by any cutaneous or motor branches of nerves.

These anatomical dissections will provide reference material for further ultrasound studies on the location of the neuro-vascular structures in reference to superficial palmar creases.

Acknowledgments The authors did not receive any outside funding or grants in support of their research for or preparation of this work.

Conflict of interest The authors declare that they have no conflict of interest.

Open Access This article is distributed under the terms of the Creative Commons Attribution License which permits any use, distribution, and reproduction in any medium, provided the original author(s) and the source are credited. 


\section{References}

1. Anand P, Trzeciak MA (2010) The clinical application of Kaplan's cardinal line as a surface marker for the superficial palmar. Arch Hand (NY) 5(2):155-159. doi:10.1007/ s11552-009-9229-0

2. Bilge O, Pinar ĆY, Ozer ĆM, Govsa ĆF (2006) A morphometric study on the superficial palmar arch of the hand. Surg Radiol Anat 28:343-350. doi:10.1007/s00276-006-0109-9

3. Bonnel F, Vila RM (1985) Anatomical study of the ulnar nerve in the hand. J Hand Surg Br 10(2):165-168

4. Brown RA, Gelberman RH (1996) Techniques in hand surgery, 1st edn. Williams \& Wilkins, Philadelphia, pp 703-710

5. Bugbee WD, Botte MJ (1993) The relationship between palmar skin creases and osseous anatomy. Clin Orthop Relat Res 296:122-126

6. Chaynes P, Becue J, Vaysse P, Laude M (2004) Relationships of the palmar cutaneous branch of the median nerve: a morphometric study. Surg Radiol Anat 26:275-280. doi:10.1007/ s00276-004-0226-2

7. Dar H, Schmidt R, Nitowsky HM (1977) Palmar crease variants and their clinical significance: a study of newborns at risk. Pediatr Res 11(2):103-108

8. Don Griot JP, Zuidam JM, Kooten EO, Prose LP, Hage JJ (2000) Anatomic study of the ramus communicans between the ulnar and median nerves. J Hand Surg 25A:948-954

9. Doyle JM, Botte JM (2002) Surgical anatomy of the hand and upper extremity, 1st edn. Lippincott Williams \& Wilkins, USA, pp 5-25

10. Ferrari GP, Gilbert A (1991) The superficial anastomosis on the palm of the hand between the ulnar and median nerves. J Hand Surg Br 16:511-514

11. Gellman H, Botte MJ, Shankwiler J (2001) Arterial patterns of the deep and superficial palmar arches. Clin Orthop Relat Res 2(383):41-46

12. Gerritsen AA, Uitdehaag BM, Geldere D, Scholten RJ, de Vet HC, Bouter LM (2002) Open Carpal tunnel release is the preferred method of surgical treatment for carpal tunnel syndrome. $\mathrm{J}$ Bone Joint Surg Am 84(8):1489

13. Hoppenfeld S, DeBoer P (1994) The wrist and hand. Surgical Exposures in orthopaedics: the anatomic approach, 2nd edn. J.B. Lippincott Co, Philadelphia, pp 147-214

14. Jelecic N, Gajisin S, Zbrodowski A (1988) Arcus palmaris superficialis. Acta Anat 132(2):187-190

15. Kaplan EB (1996) Approach to the upper extremity. Surgical approaches to the neck, cervical spine, and upper extremity, 1st edn. W.B. Saunders Co, Philadelphia, pp 143-160

16. Kaplan EB (1984) Kaplan's functional and surgical anatomy of the Hand, 5th edn. Lippincott, USA, pp 353-355

17. Kaplan EB (1953) Surface anatomy of the hand and wrist. In: Spinner E (ed) Functional and surgical anatomy of the hand, 1st edn. J.B. Lippincott Co, Philadelphia, pp 227-231
18. Kaplan EB (1968) Guide lines to deep structures and dynamics of intrinsic muscles of the hand. Surg Clin North Am 48:993-1002

19. Kimura S, Kitagawa T (1986) Embryological development of human palmar, plantar, and digital flexion creases. Anat Rec 216:191-197

20. Loukas M, Louis ZR Jr, Stewart L, Hallner B, Deluca T, Morgan W (2007) The surgical anatomy of ulnar and median nerve communications in the palmar surface of the hand. J Neurosurg 106(2):887-893

21. Martin CH, Seiler JG III, Lesesne JS (1996) The cutaneous innervations of the palm: an anatomic study of the ulnar and median nerves. J Hand Surg Am 21:634-638

22. May JW Jr, Rosen H (1981) Division of the sensory ramus communicans between the ulnar and median nerves: a complication following carpal tunnel release. J Bone Joint Surg Am 63-A(5):1036-1041

23. McLean KM, Sacks JM, Kuo YR (2008) Anatomical landmarks to the superficial and deep palmar arches. Plast Reconstr Surg 121(1):181-185. doi:10.1097/01.prs.0000293863.45614.f9

24. Meals RA, Shaner M (1983) Variations in digital sensory patterns: a study of the ulnar nerve-median nerve palmar communicating branch. J Hand Surg Am 8:411-414

25. Mumford J, Morecraft R, Blair WF (1987) Anatomy of the thenar branch of the median nerve. J Hand Surg Am 12:361-365

26. Palmer AK, Tolvonen DA (1999) Complications of endoscopic and open carpal tunnel release. J Hand Surg 24A(3):561-565

27. Riordan DC, Kaplan EB (1984) Surface anatomy of the hand and wrist. In: Spinner M (ed) Kaplan's functional and surgical anatomy of the hand, 3rd edn. J.B. Lippincott Co, Philadelphia, pp 353-360

28. Schaumann BA, Kimura S (1991) Palmar, plantar, and digital flexion creases: morphologic and clinical considerations. Birth Defects 2(27):229-252

29. Schmidt HM, Lanz U (2004) Surgical anatomy of the hand, 4th edn. Thieme, Stuttgart, pp 9-44, 85-113, 115-127, 129-172

30. Sirasanagandla SR, Patil J, Potu BK, Surekha SN, Shetty D, Bhat KM (2013) A rare anatomical variation of the Berrettini anastomosis and third common palmar digital branch of the median nerve. Anat Sci Int 88(3):163-166

31. Tsuruta T, Syed SA, Tsai T (1994) Comparison of proximal and distal one portal entry techniques for endoscopic carpal tunnel release. A cadaver study. J Hand Surg 19B(4):618-621

32. Vella JC, Hartigan BJ, Stern PJ (2006) Kaplan's cardinal line. J Hand Surg Am 31(6):912-918

33. Watchmaker GP, Weber D, Mackinnon SE (1996) Avoidance of transaction of the palmar cutaneous branch of median nerve in carpal tunnel release. J Hand Surg Am 21:644-650

34. Zancolli EA, Cozzi EP (1991) Atlas of surgical anatomy of the hand. Churchill-Livingstone, New York 